Print ISSN: 2288-4637 / Online ISSN 2288-4645

doi:10.13106/jafeb.2020.vol7.no5.009

\title{
Stock Market Response to Elections: An Event Study Method
}

\author{
Kavita CHAVALI', Mohammad ALAM ${ }^{2}$, Shireen ROSARIO ${ }^{3}$ \\ Received: March 01, 2020 Revised: March 21, 2020 Accepted: April 03, 2020
}

\begin{abstract}
The research paper examines the influence of elections on the stock market. The study analyses whether the market reaction would be the same when a party wins and comes to power for the second consecutive time. The study employs Market Model Event study methodology. The sample period taken for the study is 2014 to 2019. A sample of 31 companies listed in Bombay Stock Exchange is selected at random for the purpose of the study. For the elections held in 2014, an event window of 82 days was taken with 39 days prior to the event and 42 days post event. The event ( $\mathrm{t} 0$ ) being the declaration of the election results. For the elections held in 2019 an event window of 83 days was taken with 41 days prior to the event and 41 days post event. The results indicate that the market reacts positively with significantly positive Average Abnormal Returns. The findings of the study reveal that the impact on the market is not the same between any two elections even when the same party comes to power for the second time. The semi-strong form of efficient market hypothesis holds true in the context of emerging markets like India.
\end{abstract}

Keywords : Financial Economics, Event study, Elections, Emerging Markets, Politics

JEL Classification Code : G41, D53, H11, D91

\section{Introduction}

The impact of politics on the stock market is a debated topic from times immemorial. India is one of the emerging economies with the largest democracy in the world, which operates in accordance with the constitution of 1950. India being the second most populous country in the world has the largest number of voters eligible to take part in elections. In any democracy the elected officials are accountable to

${ }^{1}$ First Author and Corresponding Author. Associate Professor, College of Commerce and Business Administration, Dhofar University, Sultanate of Oman [Postal Address: P.O Box. 2509, Salalah, Sultanate of Oman, 211]. Email: kchavali@du.edu.om ${ }^{2}$ Assistant Professor, College of Commerce and Business Administration, Dhofar University, Sultanate of Oman.

Email:malam@du.edu.om

${ }^{3}$ Lecturer, College of Commerce and Business Administration,

Dhofar University, Sultanate of Oman. Email: srosario@du.edu.om

(C) Copyright: The Author(s)

This is an Open Access article distributed under the terms of the Creative Commons Attribution Non-Commercial License (http://Creativecommons.org/licenses/by-nc/4.0/) which permits unrestricted noncommercial use, distribution, and reproduction in any medium, provided the original work is properly cited. the people, and to continue in office they must be elected at prescribed intervals. For that reason, as per the Constitution of India, General Elections are held every five years and an elected party comes to power. Nordhaus (1975) was one of the pioneers to study of impact of politics on the stock market. The influence of politics on the stock market is explained with the semi-strong form of market efficiency hypothesis (Fama, 1970). They are called event studies (Fama, 1991).

In an event study, we can understand and measure how swiftly security prices react to announcements of election results in politics. In semi-strong Efficient Market Hypothesis, the existing market prices of stocks reflect all information publicly available. In semi-strong form of market efficiency, the market takes some time to reflect the information in price. The time taken to reflect depends on the market, on the stock and the way in which information is shared. The stock market reacts to the most possible or likely outcome of the election, even before the election day (Foster, 2012). This study attempts to check if there is a presence of semi-strong form of EMH in the Indian stock market. The authors in this study made an effort to observe the influence of politics on the stock market and its effect on the Average Abnormal Return on various stocks. 


\section{Literature Review}

There is an abundance of studies in the past, which dwelled into the influence of politics on the stock markets on economies around the world (Angela \& Wilson, 2007; Bialkowski, Gottschalk, \& Wisniewski, 2008; Chavali \& Zahid, 2011; Hung, 2011; Kumar, Sophia, \& Maria, 2015; Liu, 2007; Wong \& Hooy, 2016). The pioneers who investigated the political angle of the financial markets in academic studies are Booth and Booth (2003) and SantaClara and Valkanov (2003). The reason for the impact of politics and elections on stock market is probably because of the expectation in the changes in the government policies which the newly elected party or person might bring in, which might impact tax rates and thereby the corporate performance. The effect on stock markets depends on policy changes which the individual party or person who comes to power carry out and its impact on reaction and sentiments of the market (Wang, Lee, \& Ling, 2008)

Governments usually engage in increased consumption, increased spending in projects with high visibility prior to elections which can cause inflation. The anticipation of policy changes and increased uncertainty result in change of investors or market sentiments during the election period (Siokis \& Kapopoulos, 2007). According to Leblang and Mukherjee (2005) there are numerous ways how financial markets react to elections. Lee and Brahmasrene (2018) argue that there is no evidence of impact of global factors on the stock markets. They argue that it is the impact of macro-economic variables on stock market and stock prices in the short run rather than long run. They observed a positive relationship between macro variables and stock prices. Hong (2016) has a contrasting view and in his study argue that lack of availability of information otherwise called as information asymmetry hinders the stock prices to respond to economic conditions. The stock market volatility is because of the increase or decrease in the demand of shares when the supply is difficult to adjust in turn which alters stock prices.

Stocks become more volatile under the party that supports social equality and egalitarianism and when the market expects that party to win elections. Second, the expectations of inflation is low with a party who supports social or economic conservatism which increases demand for stocks and stimulates higher trading volume. This also leads to higher stock price volatility and higher mean. Nguyen and Nguyen (2019) in their study state that volatility increases the attractiveness of stock market for investors. Investors do not react to good and bad news in the same way. The impact on the stock market is higher for bad news compared to the good news. As per their study, it is observed that the volatility is higher when the investors perceive the news as not so good compared to good news.
Gomez and Jomo (1999) recognize the presence of a relationship between politics and companies. As per their study on an average company, which has political connections, it does better and significantly benefits during elections. It is also observed that the companies owned by Government react more to election results than private companies. This could be because during elections, politicians use companies owned by Government for political purposes (Dinc, 2005).

Huang (1985) examined and established a relation between stock market return and elections which is statistically significant. The impact on the stock markets is not the same and depends on the party that comes into power. As per their study, the stock market returns are bad in the first and second year after an election and improve in the third and fourth year. Returns are bad in the first two years because the market does not like uncertainty, which a new party brings. Siokis and Kapopoulos (2003) argues that if there are clear distinct ideological differences between the political parties in any country around the world those countries are perfect to examine the effect of General elections on financial markets. India falls into this category as there are vast differences in the ideologies of various parties. There are studies which examined the influence of elections on the stock market but studies have not explored the comparison of two General Elections in India and whether the impact on the Average Abnormal return on stocks would be the same when the same party comes to power for the second time. This study thus attempts to fill the gap by using event study methodology as an approach to examine Average Abnormal Return (AAR) and Cumulative Average Abnormal Return (CAAR) under different elections.

\section{Research Methods and Materials}

A sample of thirty one companies are selected at random for the research study. The data related to stock prices are taken from the Bombay Stock Exchange (BSE). The time period selected for the study was 20014 to 2019 (16th General Elections - 18th March 2014 to 15th July 2014 and 17th General Elections - 20th March 2019 to 23rd July 2019). The BSE 30 index closing prices for the respective period are taken. The event date is the day the result of the General Elections was declared, i.e. 16 May in 2014 and 23 May in 2019, which is taken as t0. This study assumes that the information was known to the market on the event day or Election Day. In the 16th General Elections, the stock price of a total of 82 days are taken 39 days prior to the event day, event day ( $\mathrm{t} 0$ ) and 42 days post-election and the event day. In the 17th General Elections, the stock price of a total of 83 days are taken 41 days prior to the election results, event day (t0) and 41 days post-election.

The Market Model Event study methodology is used in this study. The market model used by Fama, Fisher, Jensen, and Roll (1969) assumes a linear relationship between the 
return of the security to the return of the market portfolio. This model unlike index model and Capital Asset Pricing model is free from criticisms and detects abnormal returns better than other methodologies. Market model is proven to yield valid results (Salamudin, Ariff, \& Nassir, 1999). Market model methodology is a relatively powerful methodology applicable under wide variety of situations (Brown \& Warner, 1985).

The study conducted uses daily prices of 82 trading days for 16th General Election and 83 days for 17th General Election is used for calculation of the regression coefficients for the sample companies. A 82 day event window, t-39 to $\mathrm{t}+42$ in the 16th General Elections and 83 event window $\mathrm{t}-41$ to $\mathrm{t}+41$ in the 17th General Elections where event date ( $\mathrm{t} 0$ ) is the announcement of election results for calculating the abnormal returns (AR) the average abnormal returns (AAR) of the sample of 31 companies and the cumulative average abnormal returns (CAAR) around the An date of stocks in the sample. A comparison of Pre-election, Election and Postelection $\mathrm{AAR} \%$ returns and $\mathrm{T}$ statistics are calculated and compared for 16th and 17th General Elections.

The study undertakes to check if there is a positive average return around General Elections dates. The study examines the extent of influence of General Elections on the Indian stock market and the impact on the market sentiments between two elections would be the same when the same party comes to power for the second time.

Null hypothesis (H0): Average Abnormal Return AAR around the election result date is positive and is statistically significant

Alternate hypothesis (Ha): Average Abnormal Return AAR around the election result date is negative and is statistically significant

Table 1 shows the list of sample companies taken from BSE to test the impact of elections on stock market.

Table 2 shows the calculated regression coefficients $\alpha$ and $\beta$ values of the sample companies of 16th and 17th General Elections.

\section{Results and Discussion}

Table 3 presents results for each of the 82 days' event window, 39 pre-election announcements, and 42 post-election announcements for the entire sample of 31 companies of the General Elections held in 2014. Table 3 reports average daily AR, AARs, CARs and CAAR for the days $t-39$ to $t+41$ along with the test statistics obtained using the MS Excel descriptive analysis tool for testing the null hypothesis. The AARs are negative on the days $\mathrm{t}-37, \mathrm{t}-34, \mathrm{t}-33, \mathrm{t}-18, \mathrm{t}-15, \mathrm{t}-$ $12, \mathrm{t}-10, \mathrm{t}-9, \mathrm{t}-9, \mathrm{t}-8, \mathrm{t}-4, \mathrm{t}-1 \mathrm{t} 0, \mathrm{t} 1, \mathrm{t} 3, \mathrm{t} 6, \mathrm{t} 8, \mathrm{t} 11$ However, it is observed that there are significantly positive AARs on most of the post-election result announcement days.
CAAR is also seen to be significantly positive in the event window. This leads to acceptance of the null hypothesis (H0). This shows that the announcement of election results did boost for majority of the return of stocks in the sample. Indian National Congress party was in power in India for 49 long years since Independence and in the 16th General Elections in 2014 for the first time a new party (Bharatiya Janata Party) came into power. This positive market reaction in post-election can be attributed to the rapid absorption of the information and change in the party in power which is

Table 1: List of sample companies

\begin{tabular}{|c|c|}
\hline Company Name & Industry \\
\hline Lupin Ltd & Pharmaceutical \\
\hline Wipro Ltd & Pharmaceutical \\
\hline Tata Steel Ltd & Manufacturing \\
\hline Tata Motors Ltd & Automobile \\
\hline Tata Motors DVR Ordinary & Manufacturing \\
\hline Tata Consultancy Services Ltd & Information Technology \\
\hline Sun Pharmaceutical India Ltd & Healthcare \\
\hline State Bank of India & Banking \\
\hline Power Grid Corporation & Power \\
\hline ONGC Ltd & Oil \& Gas \\
\hline NTPC Ltd & Power \\
\hline Maruti Suzuki Ltd & Automobile \\
\hline Mahindra \& Mahindra Ltd & Automobile \\
\hline L\&T Ltd & Manufacturing \\
\hline Kotak Mahindra Bank Ltd & Banking \\
\hline ITC Ltd & FMCG \\
\hline Infosys Ltd & Information Technology \\
\hline Cipla & Healthcare \\
\hline ICICI Bank Ltd & Banking \\
\hline HDFC & Finance \\
\hline Hindustan Unilever Ltd & FMCG \\
\hline Hero Motor Corp & Automobile \\
\hline HDFC Bank Ltd & Banking \\
\hline Dr Reddy Labs Ltd & Healthcare \\
\hline Coal India Ltd & Mining \\
\hline Bharati Airtel Ltd & Telecom \\
\hline Bajaj Auto Ltd & Automobiles \\
\hline AXIS Bank & Banking \\
\hline Asian Paints Ltd & Manufacturing \\
\hline Adani Ports \& SEZ Ltd & Infrastructure \\
\hline Reliance Industries & Oil \& Gas \\
\hline
\end{tabular}


Table 2: Firm characteristics regression coefficients using market model

\begin{tabular}{|c|c|c|c|c|}
\hline \multirow[t]{2}{*}{ Company Name } & \multicolumn{2}{|c|}{$16^{\text {th }}$ General Elections 2014} & \multicolumn{2}{|c|}{ 17 $7^{\text {th }}$ General Elections 2019} \\
\hline & $\alpha$ & $\beta$ & $\alpha$ & $\beta$ \\
\hline Lupin Ltd & 0.0018 & -0.048 & 0.0002 & 0.573 \\
\hline Wipro Ltd & $-4.1 \mathrm{E}-05$ & 0.108 & 0.0004 & 0.258 \\
\hline Tata Steel Ltd & 0.0037 & 1.235 & -0.001 & 1.436 \\
\hline Tata Motors Ltd & -0.0001 & 1.147 & -0.0013 & 1.862 \\
\hline Tata Motors DVR Ordinary & 0.0029 & 1.697 & -0.0016 & 1.832 \\
\hline Tata Consultancy Services Ltd & 0.0014 & 0.091 & 0.0006 & 0.475 \\
\hline Sun Pharmaceutical India Ltd & 0.0028 & 0.0592 & -0.0007 & 1.028 \\
\hline State Bank of India & 0.0019 & 1.785 & 0.0017 & 1.621 \\
\hline Power Grid Corporation & 0.0015 & 1.323 & 0.0007 & 0.521 \\
\hline ONGC Ltd & -0.0005 & 1.916 & -0.0007 & 0.9743 \\
\hline NTPC Ltd & 0.0006 & 1.262 & $-6.2 \mathrm{E}-06$ & 0.7734 \\
\hline Maruti Suzuki Ltd & 0.0022 & 1.213 & -0.0015 & 1.4210 \\
\hline Mahindra \& Mahindra Ltd & 0.0003 & 0.772 & -0.0022 & 1.2146 \\
\hline L\&T Ltd & 0.0005 & 1.583 & 0.0006 & 1.2712 \\
\hline Kotak Mahindra Bank Ltd & 0.0009 & 0.863 & 0.0013 & 0.8954 \\
\hline ITC Ltd & -0.0006 & 0.334 & -0.0011 & 0.6224 \\
\hline Infosys Ltd & -0.001 & 0.365 & 0.0011 & 0.3135 \\
\hline Cipla & 0.0013 & 0.163 & $3.5 \mathrm{E}-05$ & 0.7980 \\
\hline ICICI Bank Ltd & -0.0005 & 1.566 & 0.0006 & 1.3761 \\
\hline HDFC & -0.0003 & 1.070 & 0.0011 & 1.1894 \\
\hline Hindustan Unilever Ltd & 0.0009 & 0.252 & 4.0E-05 & 0.5747 \\
\hline Hero Motor Corp & $-8.61 \mathrm{E}-05$ & 1.139 & -0.0004 & 1.2600 \\
\hline HDFC Bank Ltd & -0.0003 & 1.022 & 9.4E-05 & 0.9298 \\
\hline Dr Reddy Labs Ltd & $4.28 \mathrm{E}-05$ & -0.255 & -0.0003 & -0.0989 \\
\hline Coal India Ltd & 0.0013 & 1.619 & -0.0011 & 0.5245 \\
\hline Bharati Airtel Ltd & 4.67E-06 & 0.850 & -0.0008 & 1.0159 \\
\hline Bajaj Auto Ltd & -0.0003 & 0.569 & -0.0018 & 0.6677 \\
\hline AXIS Bank & 0.0021 & 1.351 & -0.0004 & 1.1294 \\
\hline Asian Paints Ltd & 0.0006 & 0.679 & $-8.1 \mathrm{E}-05$ & 0.7352 \\
\hline Adani Ports \& SEZ Ltd & 0.0012 & 2.364 & 0.0012 & 1.1614 \\
\hline Reliance Industries & -0.0016 & 1.500 & -0.0007 & 1.1735 \\
\hline
\end{tabular}

perceived positively by the market and reflected in the stock prices.

Table 4 shows the results for each of the 83 days' event window, 41 pre-election announcements, and 41 postelection announcements for the entire sample of 31 companies of the 17th General Elections conducted in 2019. It reports the average daily AR, AARs, CARs and CAAR for the days $t-41$ to $t+41$ along with the test statistics obtained using the MS Excel descriptive analysis tool for testing the null hypothesis. The AARs are negative on the days $\mathrm{t}-41$,t$39, \mathrm{t}-38, \mathrm{t}-37, \mathrm{t}-36, \mathrm{t}-32, \mathrm{t}-30, \mathrm{t}-23, \mathrm{t}-12, \mathrm{t}-9, \mathrm{t}-6, \mathrm{t}-4, \mathrm{t}-3, \mathrm{t} 3, \mathrm{t} 4, \mathrm{t} 5$ $\mathrm{t} 6, \mathrm{t} 8, \mathrm{t} 10, \mathrm{t} 11, \mathrm{t} 13, \mathrm{t} 15, \mathrm{t} 18$ However, it is observed that there are significantly positive AARs on most of the postelection result announcement days. CAAR is also seen to be significantly positive in the event window. The null hypothesis (H0) is accepted. It indicates that post-election 
Table 3: Daily Average AR and t statistics of 16th General Elections (2014) as per Market Model

\begin{tabular}{|c|c|c|c|c|c|}
\hline Day t & AAR $\%$ & T stat & Null hypothesis test & CAR\% & CAAR $\%$ \\
\hline-39 & 30.154 & 0.8416 & Can't Reject Ho & 9.35 & 30.15 \\
\hline-38 & 16.355 & 0.5042 & Can't Reject Ho & 5.07 & 46.51 \\
\hline-37 & -20.330 & -0.916 & Can't Reject Ho & -6.30 & 26.18 \\
\hline-36 & 11.196 & 0.4130 & Can't Reject Ho & 3.47 & 37.37 \\
\hline-35 & 12.327 & 1.5516 & Can't Reject Ho & 3.82 & 49.70 \\
\hline-34 & -19.434 & -0.692 & Can't Reject Ho & -6.02 & 30.27 \\
\hline-33 & 29.494 & 1.3216 & Can't Reject Ho & 9.14 & 59.76 \\
\hline-32 & -2.280 & -0.081 & Can't Reject Ho & -0.71 & 57.48 \\
\hline-31 & 15.788 & 0.6910 & Can't Reject Ho & 4.89 & 73.27 \\
\hline-30 & 30.567 & 1.5974 & Can't Reject Ho & 9.48 & 103.84 \\
\hline-29 & 11.129 & 0.4101 & Can't Reject Ho & 3.45 & 114.97 \\
\hline-28 & 15.586 & 0.5371 & Can't Reject Ho & 4.83 & 130.55 \\
\hline-27 & 19.570 & 0.7862 & Can't Reject Ho & 6.07 & 150.12 \\
\hline-26 & 5.093 & 0.2357 & Can't Reject Ho & 1.58 & 155.22 \\
\hline-25 & -1.803 & -0.107 & Can't Reject Ho & -0.56 & 153.41 \\
\hline-24 & 7.397 & 0.3779 & Can't Reject Ho & 2.29 & 160.81 \\
\hline-23 & 19.781 & 0.5832 & Can't Reject Ho & 6.13 & 180.59 \\
\hline-22 & 30.277 & 0.9954 & Can't Reject Ho & 9.39 & 210.87 \\
\hline-21 & 20.362 & 1.1130 & Can't Reject Ho & 6.31 & 231.23 \\
\hline-20 & -0.178 & -0.005 & Can't Reject Ho & -0.06 & 231.05 \\
\hline-19 & 19.615 & 0.6324 & Can't Reject Ho & 6.08 & 250.67 \\
\hline-18 & -3.409 & -0.143 & Can't Reject Ho & -1.06 & 247.26 \\
\hline-17 & 2.159 & 0.0677 & Can't Reject Ho & 0.67 & 249.42 \\
\hline-16 & -6.497 & -0.350 & Can't Reject Ho & -2.01 & 242.92 \\
\hline-15 & -17.188 & -0.886 & Can't Reject Ho & -5.33 & 225.73 \\
\hline-14 & 10.309 & 0.3681 & Can't Reject Ho & 3.20 & 236.04 \\
\hline-13 & 7.565 & 0.3249 & Can't Reject Ho & 2.35 & 243.61 \\
\hline-12 & -25.262 & -1.208 & Can't Reject Ho & -7.83 & 218.34 \\
\hline-11 & 2.715 & 0.1301 & Can't Reject Ho & 0.84 & 221.06 \\
\hline-10 & -7.308 & -0.347 & Can't Reject Ho & -2.27 & 213.75 \\
\hline-9 & -9.372 & -0.464 & Can't Reject Ho & -2.91 & 204.38 \\
\hline-8 & -18.227 & -0.927 & Can't Reject Ho & -5.65 & 186.15 \\
\hline-7 & 10.839 & 0.4710 & Can't Reject Ho & 3.36 & 196.99 \\
\hline-6 & 3.600 & 0.2512 & Can't Reject Ho & 1.12 & 200.59 \\
\hline-5 & -1.335 & -0.058 & Can't Reject Ho & -0.41 & 199.26 \\
\hline-4 & -14.566 & -0.436 & Can't Reject Ho & -4.52 & 184.69 \\
\hline-3 & 13.194 & 0.3697 & Can't Reject Ho & 4.09 & 197.88 \\
\hline-2 & 35.672 & 0.9876 & Can't Reject Ho & 11.06 & 233.56 \\
\hline-1 & -7.960 & -0.252 & Can't Reject Ho & -2.47 & 225.60 \\
\hline 0 & -7.15 & -0.158 & Can't Reject Ho & -2.22 & 218.44 \\
\hline 1 & -5.239 & -0.067 & Can't Reject Ho & -1.62 & 213.20 \\
\hline
\end{tabular}




\begin{tabular}{|c|c|c|c|c|c|}
\hline 2 & 7.502 & 0.1784 & Can't Reject Ho & 2.33 & 220.71 \\
\hline 3 & -1.529 & -0.051 & Can't Reject Ho & -0.47 & 219.18 \\
\hline 4 & 5.651 & 0.1641 & Can't Reject Ho & 1.75 & 224.83 \\
\hline 5 & 25.969 & 0.7215 & Can't Reject Ho & 8.05 & 250.80 \\
\hline 6 & -25.448 & -0.687 & Can't Reject Ho & -7.89 & 225.35 \\
\hline 7 & 4.728 & 0.2224 & Can't Reject Ho & 1.47 & 230.08 \\
\hline 8 & -15.540 & -0.532 & Can't Reject Ho & -4.82 & 214.54 \\
\hline 9 & 21.709 & 0.6864 & Can't Reject Ho & 6.73 & 236.25 \\
\hline 10 & 85.441 & 1.8576 & Can't Reject Ho & 26.49 & 321.69 \\
\hline 11 & -20.304 & -0.6846 & Can't Reject Ho & -6.29 & 301.38 \\
\hline 12 & 3.895 & 0.1039 & Can't Reject Ho & 1.21 & 305.28 \\
\hline 13 & 26.756 & 1.0609 & Can't Reject Ho & 8.29 & 332.04 \\
\hline 14 & -0.505 & -0.018 & Can't Reject Ho & -0.16 & 331.53 \\
\hline 15 & 41.473 & 1.0981 & Can't Reject Ho & 12.86 & 373.00 \\
\hline 16 & 52.163 & 1.2925 & Can't Reject Ho & 16.17 & 425.17 \\
\hline 17 & 15.214 & 0.5049 & Can't Reject Ho & 4.72 & 440.38 \\
\hline 18 & 12.577 & 0.3938 & Can't Reject Ho & 3.90 & 452.96 \\
\hline 19 & 10.863 & 0.4042 & Can't Reject Ho & 3.37 & 463.82 \\
\hline 20 & -36.040 & -1.649 & Can't Reject Ho & -11.17 & 427.78 \\
\hline 21 & -16.785 & -0.691 & Can't Reject Ho & -5.20 & 410.99 \\
\hline 22 & 10.282 & 0.4805 & Can't Reject Ho & 3.19 & 421.28 \\
\hline 23 & 30.313 & 1.2527 & Can't Reject Ho & 9.40 & 451.59 \\
\hline 24 & 15.818 & 0.4121 & Can't Reject Ho & 4.90 & 467.41 \\
\hline 25 & 18.664 & 1.0143 & Can't Reject Ho & 5.79 & 486.07 \\
\hline 26 & 33.677 & 0.9305 & Can't Reject Ho & 10.44 & 519.75 \\
\hline 27 & -36.113 & -1.953 & Reject Ho & -11.20 & 483.64 \\
\hline 28 & 20.544 & 0.6784 & Can't Reject Ho & 6.37 & 504.18 \\
\hline 29 & 20.849 & 0.8620 & Can't Reject Ho & 6.46 & 525.03 \\
\hline 30 & 30.337 & 0.8674 & Can't Reject Ho & 9.40 & 555.36 \\
\hline 31 & 0.328 & 0.0129 & Can't Reject Ho & 0.10 & 555.69 \\
\hline 32 & 14.557 & 0.4735 & Can't Reject Ho & 4.51 & 570.25 \\
\hline 33 & 33.389 & 1.0479 & Can't Reject Ho & 10.35 & 603.64 \\
\hline 34 & -0.949 & -0.040 & Can't Reject Ho & -0.29 & 602.69 \\
\hline 35 & -1.899 & -0.112 & Can't Reject Ho & -0.59 & 600.79 \\
\hline 36 & 7.226 & 0.2799 & Can't Reject Ho & 2.24 & 608.02 \\
\hline 37 & -36.867 & -1.407 & Can't Reject Ho & -11.43 & 571.15 \\
\hline 38 & -26.918 & -0.977 & Can't Reject Ho & -8.34 & 544.23 \\
\hline 39 & 1.841 & 0.0834 & Can't Reject Ho & 0.57 & 546.07 \\
\hline 40 & 4.388 & 0.1456 & Can't Reject Ho & 1.36 & 550.46 \\
\hline 41 & 22.465 & 0.8201 & Can't Reject Ho & 6.96 & 572.93 \\
\hline 42 & 6.570 & 0.2593 & Can't Reject Ho & 2.04 & 579.50 \\
\hline
\end{tabular}


Table 4: Daily Average AR and t statistics of 17th General Elections (2019) as per Market Model

\begin{tabular}{|c|c|c|c|c|c|}
\hline Day $t$ & AAR $\%$ & T stat & Reject/Accept & CAR & CAAR \\
\hline-41 & -0.570 & -2.1181 & Reject Ho & -17.678 & -0.570 \\
\hline-40 & 0.109 & 0.4778 & Can't Reject Ho & 3.369 & -0.462 \\
\hline-39 & -0.118 & -0.4855 & Can't Reject Ho & -3.673 & -0.580 \\
\hline-38 & -0.198 & -1.0064 & Can't Reject Ho & -6.144 & -0.778 \\
\hline-37 & -0.380 & -2.5089 & Can't Reject Ho & -11.790 & -1.159 \\
\hline-36 & -0.237 & -0.8605 & Can't Reject Ho & -7.346 & -1.396 \\
\hline-35 & 0.293 & 1.6522 & Can't Reject Ho & 9.075 & -1.103 \\
\hline-34 & 0.513 & 1.3251 & Can't Reject Ho & 15.894 & -0.590 \\
\hline-33 & 1.320 & 3.2362 & Can't Reject Ho & 40.914 & 0.730 \\
\hline-32 & -0.530 & -2.9011 & Reject Ho & -16.427 & 0.200 \\
\hline-31 & 0.018 & 0.0866 & Can't Reject Ho & 0.566 & 0.218 \\
\hline-30 & -0.116 & -0.6118 & Can't Reject Ho & -3.592 & 0.102 \\
\hline-29 & 0.035 & 0.1975 & Can't Reject Ho & 1.077 & 0.137 \\
\hline-28 & 0.213 & 0.8280 & Can't Reject Ho & 6.596 & 0.350 \\
\hline-27 & 0.775 & 2.4060 & Can't Reject Ho & 24.034 & 1.125 \\
\hline-26 & 0.151 & 0.8189 & Can't Reject Ho & 4.692 & 1.276 \\
\hline-25 & 0.112 & 0.5567 & Can't Reject Ho & 3.484 & 1.389 \\
\hline-24 & 0.702 & 1.7484 & Can't Reject Ho & 21.749 & 2.090 \\
\hline-23 & -0.196 & -0.9878 & Can't Reject Ho & -6.089 & 1.894 \\
\hline-22 & 0.127 & 0.5885 & Can't Reject Ho & 3.926 & 2.021 \\
\hline-21 & 0.496 & 2.7846 & Can't Reject Ho & 15.385 & 2.517 \\
\hline-20 & 0.039 & 0.1293 & Can't Reject Ho & 1.213 & 2.556 \\
\hline-19 & -0.678 & -2.3051 & Reject Ho & -21.016 & 1.878 \\
\hline-18 & 0.260 & 1.6540 & Can't Reject Ho & 8.064 & 2.138 \\
\hline-17 & -0.443 & -1.2868 & Can't Reject Ho & -13.729 & 1.695 \\
\hline-16 & -0.068 & -0.2769 & Can't Reject Ho & -2.118 & 1.627 \\
\hline-15 & -0.193 & -0.7088 & Can't Reject Ho & -5.985 & 1.434 \\
\hline-14 & 0.096 & 0.4486 & Can't Reject Ho & 2.986 & 1.530 \\
\hline-13 & 0.022 & 0.1093 & Can't Reject Ho & 0.680 & 1.552 \\
\hline-12 & -0.109 & -0.4020 & Can't Reject Ho & -3.387 & 1.443 \\
\hline-11 & 0.034 & 0.2362 & Can't Reject Ho & 1.060 & 1.477 \\
\hline-10 & 0.137 & 0.6385 & Can't Reject Ho & 4.234 & 1.614 \\
\hline-9 & -0.095 & -0.3239 & Can't Reject Ho & -2.950 & 1.519 \\
\hline-8 & -0.662 & -1.8576 & Reject Ho & -20.507 & 0.857 \\
\hline-7 & 0.170 & 0.5546 & Can't Reject Ho & 5.275 & 1.027 \\
\hline-6 & -0.817 & -2.5988 & Reject Ho & -25.315 & 0.211 \\
\hline-5 & 0.074 & 0.3587 & Can't Reject Ho & 2.308 & 0.285 \\
\hline-4 & -0.421 & -1.3574 & Can't Reject Ho & -13.049 & -0.136 \\
\hline-3 & -0.146 & -0.4345 & Can't Reject Ho & -4.538 & -0.282 \\
\hline-2 & -0.593 & -2.0608 & Reject Ho & -18.387 & -0.875 \\
\hline-1 & 0.207 & 1.0661 & Can't Reject Ho & 6.419 & -0.668 \\
\hline
\end{tabular}




\begin{tabular}{|c|c|c|c|c|c|}
\hline 0 & 0.537 & 1.7188 & Can't Reject Ho & 16.651 & -0.131 \\
\hline 1 & 0.120 & 0.5219 & Can't Reject Ho & 3.712 & -0.011 \\
\hline 2 & 0.014 & 0.0482 & Can't Reject Ho & 0.424 & 0.002 \\
\hline 3 & -0.189 & -0.9039 & Can't Reject Ho & -5.847 & -0.186 \\
\hline 4 & -0.369 & -1.6518 & Reject Ho & -11.433 & -0.555 \\
\hline 5 & -0.250 & -1.0807 & Can't Reject Ho & -7.742 & -0.805 \\
\hline 6 & -0.007 & -0.0309 & Can't Reject Ho & -0.206 & -0.812 \\
\hline 7 & 0.243 & 0.8880 & Can't Reject Ho & 7.542 & -0.568 \\
\hline 8 & -0.006 & -0.0293 & Can't Reject Ho & -0.199 & -0.575 \\
\hline 9 & 0.416 & 1.9421 & Can't Reject Ho & 12.886 & -0.159 \\
\hline 10 & -0.516 & -2.8257 & Reject Ho & -16.007 & -0.675 \\
\hline 11 & -0.050 & -0.2352 & Can't Reject Ho & -1.544 & -0.725 \\
\hline 12 & 0.021 & 0.0957 & Can't Reject Ho & 0.643 & -0.705 \\
\hline 13 & -0.002 & -0.0116 & Can't Reject Ho & -0.056 & -0.706 \\
\hline 14 & 0.040 & 0.2853 & Can't Reject Ho & 1.255 & -0.666 \\
\hline 15 & -0.090 & -0.5286 & Can't Reject Ho & -2.800 & -0.756 \\
\hline 16 & -0.334 & -1.7773 & Reject Ho & -10.360 & -1.090 \\
\hline 17 & -0.049 & -0.2602 & Can't Reject Ho & -1.508 & -1.139 \\
\hline 18 & -0.534 & -1.7948 & Reject Ho & -16.555 & -1.673 \\
\hline 19 & 0.147 & 0.6200 & Can't Reject Ho & 4.550 & -1.526 \\
\hline 20 & 0.103 & 0.5133 & Can't Reject Ho & 3.190 & -1.423 \\
\hline 21 & -0.038 & -0.1609 & Can't Reject Ho & -1.191 & -1.462 \\
\hline 22 & 0.057 & 0.3255 & Can't Reject Ho & 1.774 & -1.405 \\
\hline 23 & 0.381 & 1.7172 & Can't Reject Ho & 11.816 & -1.023 \\
\hline 24 & 0.270 & 1.0954 & Can't Reject Ho & 8.373 & -0.753 \\
\hline 25 & -0.038 & -0.2262 & Can't Reject Ho & -1.185 & -0.792 \\
\hline 26 & 0.059 & 0.2145 & Can't Reject Ho & 1.827 & -0.733 \\
\hline 27 & -0.281 & -1.2478 & Can't Reject Ho & -8.716 & -1.014 \\
\hline 28 & -0.085 & -0.8022 & Can't Reject Ho & -2.643 & -1.099 \\
\hline 28 & 0.060 & 0.4004 & Can't Reject Ho & 1.850 & -1.039 \\
\hline 30 & -0.857 & -2.4947 & Reject Ho & -26.563 & -1.896 \\
\hline 31 & -0.528 & -2.0366 & Reject Ho & -16.371 & -2.424 \\
\hline 32 & 0.366 & 1.2729 & Can't Reject Ho & 11.350 & -2.058 \\
\hline 33 & -0.104 & -0.5124 & Can't Reject Ho & -3.211 & -2.162 \\
\hline 34 & 0.373 & 1.5735 & Can't Reject Ho & 11.551 & -1.789 \\
\hline 35 & 0.179 & 0.6871 & Can't Reject Ho & 5.553 & -1.610 \\
\hline 36 & -0.221 & -0.6870 & Can't Reject Ho & -6.863 & -1.832 \\
\hline 37 & 0.565 & 2.2827 & Can't Reject Ho & 17.518 & -1.266 \\
\hline 38 & -0.435 & -2.2904 & Reject Ho & -13.498 & -1.702 \\
\hline 39 & -0.728 & -2.4580 & Can't Reject Ho & -22.568 & -2.430 \\
\hline 40 & -0.173 & -0.7937 & Can't Reject Ho & -5.377 & -2.603 \\
\hline 41 & 0.769 & 2.2467 & Can't Reject Ho & 23.833 & -1.835 \\
\hline
\end{tabular}


Table 5: Comparison of AAR across different event windows for the General Elections 2014 \& 2019

\begin{tabular}{|l|c|c|c|c|}
\hline \multicolumn{5}{|c|}{ 16th General Elections 2014 } \\
\hline \multicolumn{1}{|c|}{ Event window } & AAR\% & T stat & Null hypothesis test & Significance \\
\hline Pre-Election Phase & 1.534 & 1.8142 & Reject Ho & $*$ \\
Election Phase & 0.650 & 0.6156 & Can't Reject Ho & - \\
Post-Election Phase & 3.611 & 2.5297 & Can't Reject Ho & - \\
\hline
\end{tabular}

* Significant at $5 \%$ level of significance

\begin{tabular}{|l|c|c|c|c|}
\hline \multicolumn{5}{|c|}{ 17th General Elections 2019 } \\
\hline \multicolumn{1}{|c|}{ Event window } & AAR\% & T stat & Null hypothesis test & Significance \\
\hline Pre-Election Phase & 1.519 & 1.619 & Can't Reject Ho & - \\
Election Phase & -1.650 & -1.744 & Can't Reject Ho & - \\
Post-Election Phase & -1.607 & -1.198 & Can't Reject Ho & - \\
\hline
\end{tabular}

* Significant at $5 \%$ level of significance

announcements, the returns of the stocks did boost for majority of the stocks in the sample. A new party came into power the second time in the 17th General Elections.

Table 5 shows the comparison of AAR across different event windows for the 16th and 17th General Elections. In the pre-election phase stock market reaction was different for both the elections. In the 16th General Election $\mathrm{H} 0$ was rejected at 5-percent level of significance, but during the Election and the Post-Election phase the AAR was positive and $\mathrm{H} 0$ is accepted. This could be probably because the same party was in power for 49 long years and the expectation that any other party will win and gain majority was a difficult thing to believe taking the past history of Indian politics into consideration. The AAR in the Election and the post-election stage was positive probably because the people wanted the change and the reaction is shown in the returns of stock market. In the 17th General Elections, when a new party came to power for the second consecutive time, the AAR was found positive in the pre-election stage and became negative during elections and the post-election scenario. It is observed that the stock market reaction was more intense when a party came to power for the first time than the second time.

\section{Conclusions}

India being the largest democracy in the world, politics and elections have an important role to play. The study examines the extent of influence of General Elections on the Indian stock market and the impact on the market sentiments between two elections would be the same when the same party comes to power for the second time. A healthy economy increases the chances of re-election for an incumbent candidate or party. The study reveals that there is a positive market reaction to elections in terms of $\mathrm{AAR} \%$ in case of Indian stock market. A sample of 31 BSE listed companies are taken for the study. The AAR is computed for the 82 days event window around the event day - election results during 2014 and 2019. The study finds the evidence of a positive $\mathrm{AR}$ around the election dates and confirms that elections have a positive and a favorable impact on the stock market performance of stocks in the Indian context which is in line with the previous studies. However, it is observed that the stock market reaction was more intense when a party came to power for the first time than the second time.

The study is based on two general election cycles in India. The result holds true for the select sample and during the period considered for the study. It may not be generalized for different market environments. The implications of this study are that investors can take precautionary steps before trading in stocks in the period of General Election. Riskaverse investors can avoid trading around national elections to avoid the risk linked with volatility of stocks during the elections. The result of this study will help investors to understand the stock market better and evaluate the impact of politics on stock markets during elections.

\section{References}

Angela, K., \& Ngugi, W. (2007). Stock Market Performance Before and After General Elections: A case of The Nairobi Stock Exchange (Doctoral dissertation). University of Nirobi, Nirobi, Kenya.

Bialkowski, J., Gottschalk, K., \& Wisniewski, T. P. (2008). Stock market volatility around National elections. Journal of Banking \& Finance, 32(9), 1941-1953.

Booth, J. R., \& Booth, L. C. (2003). Is presidential cycle in security returns merely a reflection of business conditions? Review of Financial Economics, 12(2), 131-159. 
Brown, S. J., \& Warner, J. B. (1985). Using daily stock returns: The case of event studies. Journal of Financial Economics, 14, 3-31.

Chavali, K., \& Zahid, Z. (2011). Impact of stock splits on stock price performance of selected companies in Indian context. Afro-Asian J. of Finance and Accounting, 2(3), 270-282.

Dinc, S. (2005). Politicians and banks: Political influences on government-owned banks in emerging countries. Journal of Financial Economics, 77(2), 453-459.

Fama, E., Fisher, L., Jensen, M. C., \& Roll, R. (1969). The adjustment of stock prices to new information. International Economic Review, 10, 1-21.

Fama, E. (1970). Efficient capital markets: a review of theory and empirical work. The Journal of Finance, 2, 383-417.

Fama, E. (1991). Efficient capital markets II. The Journal of Finance, 46, 1575-1618.

Foster, L. (2012). Does the US Presidential Election Impact the Stock Market?

Gomez, E. T., \& Jomo, K. S. (1999). Malaysia's Political Economy: Politics, Patronage and Profits. Cambridge, UK: Cambridge University Press.

Huang, R. D. (1985). Common stock returns and Presidential elections. Financial Analysts Journal, 41(2), 58-61.

Hung, L. C. (2011). The Presidential Election and the Stock Market in Taiwan. Journal of Business and Policy Research, 6(2), 36-48.

Hong, H. (2016). Information Cascade and Share Market Volatility: A Chinese Perspective. Journal of Asian Finance, Economics and Business, 3(4), 17-24. https://doi.org/10.13106/jafeb.2016. vol3.no4.17

Kumar, D. B., Sophia, S., \& Maria, E. (2015). Empirical study on Effects of the Lok Sabha Elections on stock market performance. Research Journal of Management Sciences, 14(2), 1-9.

Leblang, D., \& Mukherjee, B. (2005). Government Partisanship, Elections, and the Stock Market: Examining American and British Stock Returns, 1930-2000. American Journal of Political Science, 9(4), 780-802.
Lee, J. W., \& Brahmasrene, T. (2018). An Exploration of Dynamical Relationships between Macroeconomic Variables and Stock Prices in Korea. Journal of Asian Finance, Economics and Business, 5(3), 7-17. http://doi.org/10.13106/jafeb.2018.vol5.no3.7

Liu, L. F. (2007). An Empirical study of Presidential Elections Effect on Stock Market in Taiwan, South Korea, Singapore, Philippine, and Indonesia. Research Journal of Management Sciences, 4(2), 1-9.

Nordhaus, W. (1975). The political business cycle. Review of Economic Studies. 42(2), 169-190.

Nguyen, C. T., \& Nguyen, M. H. (2019). Modeling Stock Price Volatility: Empirical Evidence from the Ho Chi Minh City Stock Exchange in Vietnam. Journal of Asian Finance, Economics and Business, 6(3), 19-26. https://doi.org/10.13106/ jafeb.2019.vol6.no3.19

Salamudin, N., Ariff, M., \& Nassir, A.M. (1999). Economic influence on rights issue announcement behaviour in Malaysia. Pacific-Basin Finance Journal, 7(3), 405-427.

Santa-Clara, P., \& Valkanov, R. (2003). The Presidential Puzzle: Political Cycles and the Stock Market. The Journal of Finance, 58(5), 1841-1872.

Siokis, F., \& Kapopoulos, P. (2007). Parties Functions and Stock Market Volatility: Evidence from a small open economy. Economics and Politics, 19(1), 123-133.

Siokis, F., \& Kapopoulos, P. (2003). Electoral Management, Political Risk and Exchange Rate Dynamics: The Greek Experience. Applied Financial Economics, 13, 279-285.

Wong, W. Y., \& Hooy, C. W. (2016). The Impact of Election on Stock Market Returns of Government-Owned Banks: The Case of Indonesia, Malaysia and Thailand. Asian Journal of Business and Accounting, 9(1), 31-57.

Wang, Y. H., Lee, M. Y., \& Ling, C. Y. (2008). General Election, Political Change and Market Efficiency: Long and Short-Term Perspective in Developed Stock Market. Journal of Money, Investment and Banking, 9, 61-78. 